\title{
Peer reviewer data revealed
}

\author{
Although often not publicly identified, the personalities and life experiences of our referees affect their reviewing \\ practices. Therefore having the most diverse set of reviewers possible underpins our efforts to ensure a fair peer \\ review process.
}

ast month, Peer Review Week 2021

focused on 'Identity in Peer Review'.

It's a welcome theme that spotlights

the people behind the process, all working

together to maintain high-quality

publications that experts and laypersons can trust. As editors, we continually strive to improve this process, to make it as diverse, equitable and inclusive as possible. We have previously written about reducing workload and bias by using a larger pool of reviewers, offering dual anonymous review and not consulting the same reviewer more than once a year.

This time we look more closely at the personal and social identities of the reviewers themselves. Since June 2018, Nature Portfolio has been collecting voluntary data from our referees on gender, seniority and geographic location. Note that geography provides no information on citizenship or ethnicity, and that for gender the choices are 'male', 'female, 'non-binary' and 'prefer not to say'. And this way we can track how our efforts in diversifying the reviewer base are succeeding and whether we need to do more in a particular area. The gender data show that women make up $28 \%$ of our reviewers (Fig. 1a). Compared with an IAU membership of $21 \%$ women (as of August 2021; https://www.iau.org/ public/themes/member_statistics/), which is historically male-skewed, the gender ratio of our reviewer base is at least representative of the communities that we serve.

In terms of career stage, Fig. 1b shows that the majority (43\%) of our reviewers are tenured professors or researchers of similar seniority levels. According to the IAU, over-50s make up $66 \%$ of the membership, whereas only $4.1 \%$ are below 35 years of age. Thus early-career researchers represent a larger proportion of our reviewer base than they do within the community at large. These less-experienced scientists generally know the latest literature very well and are not as over-committed as the more established researchers. As we aim for three reviewers per paper, we almost always have a range of complementary perspectives. If novice reviewers need help, we provide a peer review course online (https:// masterclasses.nature.com/courses/205). We are also currently participating in a trial for

a
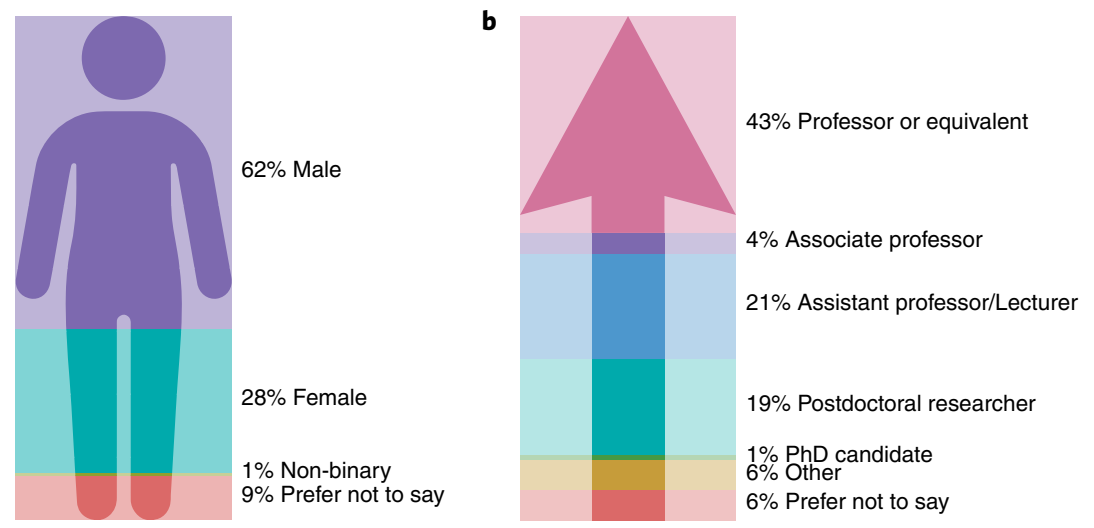

c

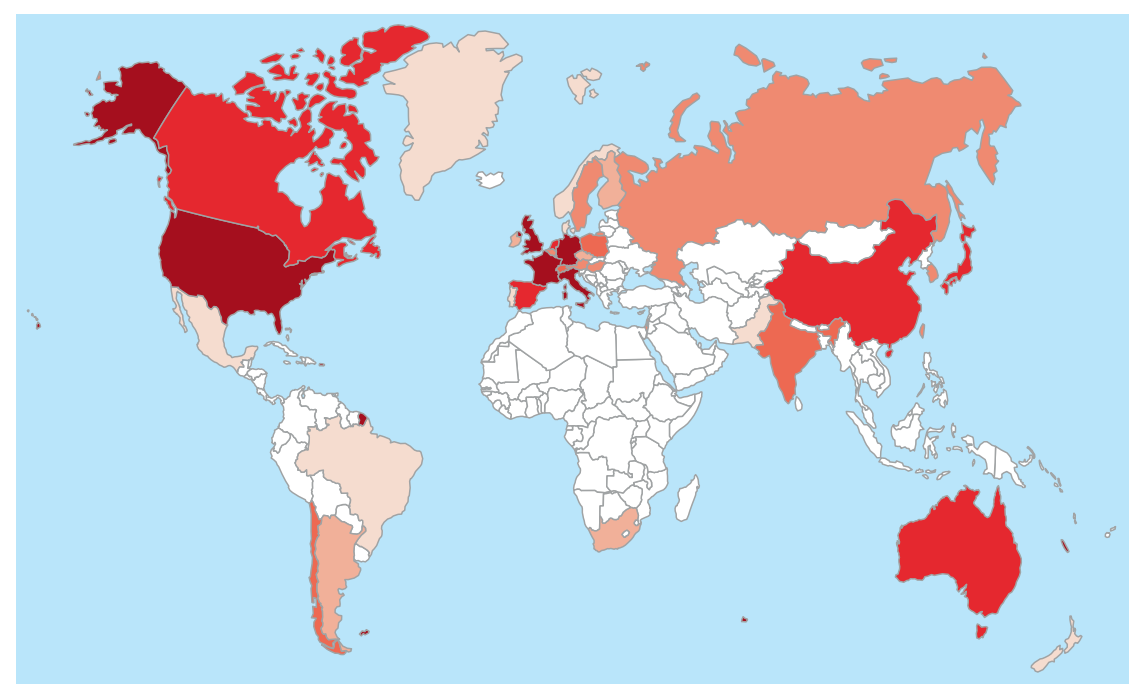

Fig. 1 | Peer reviewer data for the period June 2018 to July 2021. a, Self-reported gender distribution of reviewers. b, Reviewer seniority. c, Geographical distribution of reviewers. Dark red represents the highest count; white represents zero. Credit: Paul Woods.

mentoring early-career researchers who have not reviewed for Nature Portfolio before. These mentees review alongside seasoned experts and receive feedback from the editors.

As for geographical distribution (Fig. 1c), it is not surprising that USA and Western Europe dominate (dark red represents the highest number; white represents zero), given the funding availability for astronomical research. However, we nonetheless try to cover more of the globe. To reach new reviewers, we always ask declining reviewers to think of alternative reviewers from underrepresented communities. It would also help tremendously if institutes would provide an up-to-date and well-organized list of staff and their research interests so that we can find people ourselves. With Google translation services, English is not a concern. But information must be present and organized in a simple way. Moreover, researchers with common names benefit from having a unique persistent digital identifier (ORCID), which is why it's our 
policy for the corresponding authors to include their ORCID identifiers in their publications. Helpfully, ORCIDs can now be searched for on NASA's Astrophysics Data System, which is our go-to tool for reviewer searches.

When a paper is published, we thank the reviewers openly if they have consented to be named. Besides giving reviewers the credit that they deserve, we hope that our readers will recognize and identify with some of the names and feel encouraged that they also have a role to play, particularly if they are from a historically underrepresented group. And for the early-career researchers, the experience of reviewing for Nature Astronomy lowers the barrier when it comes to submitting their work to the Nature Portfolio journals. This positive feedback system will hopefully help us include a more diverse set of authors and reviewers in an equitable process. We will keep tracking our reviewers' gender, career stage and location distributions, and sending out surveys, to measure our progress in improving our practices and reviewer experiences.

Published online: 12 November 2021 https://doi.org/10.1038/s41550-021-01540-8 